\title{
Sentido del humor y empatía en maestras de primaria: reflexiones sobre su impacto en la enseñanza
}

Sense of humor and empathy in elementary school teachers: Reflections on its impact on teaching

Putrino Natalia

nataliaines.putrino@gmail.com

\section{Sasso Romina}

Mustaca Alba

albamustaca@gmail.com

*Facultad de Psicología y Relaciones Humanas, Universidad Abierta Interamericana, Buenos Aires, Argentina. **Centro Interdisciplinario de Investigaciones en Psicología

Matemática y Experimental (CIIPME) - Consejo Nacional de Investigaciones Científicas y Técnicas (CONICET), Buenos Aires, Argentina. DOI: 10.32654/CONCIENCIAEPG.3-1.4

Para referenciar este artículo:

Putrino, N., Sasso, R., \& Mustaca, A. (2018). Sentido del humor y empatía en maestras de primaria: reflexiones sobre su impacto en la enseñanza. REVISTA ConCiencia EPG, 3(1), 57-71. doi: https://doi.org/10.32654/

CONCIENCIAEPG.3-1.4
Fecha de recepción: 19 -02-2018 |Fecha de aceptación: 20 -06-2018

\section{Resumen}

7 objetivo de este trabajo es estudiar la relación entre los estilos de humor adaptativos y desadaptativos con la empatía cognitiva y afectiva en maestras.

Se trabajó con una muestra de 84 maestras argentinas, quienes respondieron a dos escalas: Test de empatía cognitiva y afectiva (TECA, López- Pérez, Fernández- Pinto \& Abad, 2008), y a la versión adaptada al español y en la población argentina por Cayssials y Perez (2005) de la Escala de Estilos de Humor (Martin, 2003).

Los resultados indican una relación significativa entre el tipo de humor afiliativo y las cuatro subescalas de empatía, como también entre el humor descalificación personal y el estrés empático.

Por otro lado, la dimensión adopción de perspectiva predice negativamente al humor agresivo y el estrés empático predice positivamente el humor descalificación personal.

Estos resultados se discutirán en función de estudios previos y de la posible importancia que tienen para la docencia.

Palabras clave: docentes, empatía, humor 


\section{Summary}

$\mathrm{T}$ he objective of this work is to study the relationship between Adaptive and Disadaptive humor styles with cognitive and affective empathy in teachers. We worked with a sample of 84 Argentina's teachers, who responded to two scales: Test of cognitive and affective empathy (TECA, López-Pérez, Fernández-Pinto \& Abad, 2008), and the version adapted to Spanish and in the population of Argentina by Cayssials and Perez (2005) of the Scale of Humor Styles (Martin, 2003).

The results indicate a significant relationship between the type of affiliative humor and the 4 subscales of empathy, as well as between self-defeating humor and empathic stress. On the other hand, the perspectivetaking negatively predicts aggressive mood and empathic stress positively predicts selfdefeating humor.

These results will be discussed based on previous studies and the possible importance them have for teaching.

Key words: teachers, empathy, humor

\section{Introducción}

$\mathrm{L}$

a empatía es la habilidad o capacidad para comprender los estados mentales de los otros y resonar con las emociones ajenas. El concepto no posee un consenso entre los teóricos e investigadores del área, por lo que hay varias definiciones y operacionalizaciones. En general, se la suele considerar como un constructo multimodal, donde interaccionan aspectos cognitivos y emocionales (Davis, 1980).
Los aspectos cognitivos están vinculados a la capacidad de comprender el pensamiento e intensión de los demás para poder adoptar la perspectiva del otro. Por otro lado,la dimensión emocional se asocia a la reacción ante el estado emocional de otra persona, modulada por la tendencia a imitar y sincronizar automáticamente expresiones faciales, vocalizaciones, posturas y movimientos con los de otra persona, en consecuencia, a converger emocionalmente (Hatfield, Cacioppo \& Rapson, 1994).

Otros autores hacen referencia a la tendencia a experimentar de forma vicaria (aprendizaje observacional) los estados emocionales de otros. Se trata de una compleja forma de inferencia psicológica en la que la observación, la memoria, el conocimiento y el razonamiento se combinan para poder comprender los pensamientos $\mathrm{y}$ sentimientos de los demás (Moya-Albiol, Herrero \& Bernal, 2010).

La habilidad empática o empatía se pone en juego en relación a los otros, convirtiéndose en una precursora primordial de la conducta prosocial que tiene lugar en contextos sociales complejos (Richaud, 2014). Moya-Albiol y col. (2010) destacan que en las últimas décadas se resalta la relevancia de la empatía en la disposición prosocial de las personas y su función inhibidora de la agresividad, tanto física como verbal. En ese sentido, puede ser interesante evaluarla en docentes y educadores, en general.

Para evaluar la empatía y enmarcándose en una aproximación integradora, se ha desarrollado para la población de habla hispana el Test de Empatía Cognitiva y Afectiva (TECA, LópezPérez, Fernández-Pinto y Abad, 2008). Esta escala muestra una adecuada fiabilidad y validez; 
además, dentro de las subescalas cognitivas contempla la comprensión emocional, recogiendo la distinción de Eisenberg y Strayer (1987) de adopción de perspectiva afectiva. Dentro de las facetas emocionales tiene en cuenta la empatía como respuesta emocional positiva ante un suceso vicario positivo, aspecto no contemplado previamente en otras escalas.

El test incluye dos escalas destinadas a medir la empatía cognitiva y dos escalas relacionadas con la empatía afectiva. La empatía cognitiva abarca la adopción de perspectivas (AP) y comprensión emocional (CE), por su parte, la empatía afectiva evalúa el estrés empático (EE) y alegría empática (AE).

\section{Empatía en docentes}

Los humanos aprenden a ser empáticos a partir del contacto con los otros, por aprendizaje observacional. De allí que puede pensarse que los alumnos en el ámbito escolar aprenderían en gran medida por imitación, siendo su maestro uno de los modelos de comportamiento a seguir.

Es probable que un docente empático genere simpatía y aceptación en sus alumnos y tenga los recursos necesarios para desarrollar esta capacidad en cada uno de ellos.

Para empatizar con otra persona, son fundamentales dos elementos: 1) la capacidad de comprender e identificar los sentimientos y la perspectiva de otra persona; y, 2) la capacidad de comunicar esa comprensión al individuo con el que uno se identifica (Roger, 1957).

Para Decety y Ickes (2011) la comprensión del estudiante es necesaria pero no suficiente, lo importante es la manera en que los maestros comunican a los estudiantes su comprensión de cómo se sienten los alumnos.
Investigaciones previas han mostrado que la empatía de los docentes juega un rol primordial en diferentes aspectos del aprendizaje.

Los estudiosmuestranquelos estudiantes que experimentan comunicaciones empáticas de sus maestros desarrollan una mayor comprensión y aceptación incrementando actitudes más positivas hacia ellos mismos y hacia la escolarización (Carkhuff \& Berenson, 1967; Chang, 2003).

Por otro lado, los docentes suelen expresar sensaciones de agotamiento y cansancio vinculados a la cantidad de horas laborales, pocos recursos institucionales, bajo reconocimiento social, la interacción con los alumnos y sus familias (Durán, Extremera, Montalbán \& Rey, 2005), y eso podría afectar a la predisposición empática.

\section{Estilos de Humor}

Uno de los primeros psicólogos en definir el humor ha sido Eysenck (1942), quién lo conceptualizó como el ingenio o la capacidad de las personas para interpretar las cosas de una forma divertida. Para el autor de este trabajo el humor posee tres factores: cognitivo, afectivo y conactivo.

La interacción de los tres permite la comprensión de un chiste. El afectivo está vinculado a las emociones como la alegría, el cognitivo orientado a la comprensión de la situación y el conactivo, una "adaptación superior" en la que el humor se traduce en un fenómeno adaptativo; como si la persona aprendiera a adaptarse a la situación al experimentarla de manera divertida.

El concepto de humor es desarrollado 
con interés por la corriente de la psicología positiva. Seligman (2007) considera que el humor promueve las fortalezas propias del ser humano, definiéndolo como una capacidad para experimentar una reacción muy específica como lo es la risa y de esta manera conseguir o mantener un estado de ánimo positivo.

En el mismo sentido, Illán (2015) argumenta que el humor facilita la adaptación al cambio y a salir con menos miedo de nuestra zona de confort; reduce el sentimiento de frustración que sentimos al darnos cuenta de nuestras propias limitaciones.

El humor posee varios beneficios en diferentes esferas de la vida, a partir de sus funciones. Por ejemplo, en el ámbito social posibilita compartir ideas y expresar el descontento en forma agradable, ganando una aceptación positiva que de otra forma no sería tolerada (Camacho, 2005).

Según Alemany y Cabestrero (2002) el humor facilita la recepción positiva de ideas, establecer un vínculo, fortalecer las relaciones, como en la esfera sexual. Asimismo, permite relajar las tensiones, reducir la distancia entre las personas, reforzar el sentido de confianza y persuadir a los demás.

Se ha desarrollado una medida que distingue entre usos potencialmente beneficiosos o perjudiciales del humor. Martin et al., (2003) crearon el Cuestionario de Estilos de Humor (HSQ- Humor Styles Questionnaire) que evalúan cuatro estilos de humor, dos de los cuales son hipotéticamente benéficos para el bienestar psicosocial (afiliativo y mejoramiento personal) y dos potencialmente perjudiciales (descalificación personal y agresividad).
El afiliativo es una forma de humor interpersonal que involucra contar chistes, decir cosas graciosas o bromas ingeniosas para tranquilizar o divertir a los demás y mejorar las relaciones.

El mejoramiento personal se refiere a la tendencia a mantener una visión humorística de la vida incluso en momentos de estrés, y a utilizar el humor como una estrategia de afrontamiento.

Por el contrario, el agresivo se refiere a la predisposición a usar el humor para atacar o humillar a otras personas, y por lo tanto implica sarcasmo, burlas, hostilidad o desprecio.

Finalmente, el humor descalificación
personal implica uno excesivamente
autodespreciativo, con intentos de entretener a los
demás haciendo o diciendo cosas graciosas a costa
de uno como forma de congraciarse u obtener
aprobación, permitiéndose ser el "blanco" de los
demás, y reírse junto con otros al ser ridiculizados
o menospreciados (Martin et al., 2003).

\section{El humor en el contexto educativo}

Las investigaciones previas han mostrado que los estudiantes suelen considerar el "sentido del humor" como una de las características más valoradas en un buen docente (Jáuregui \& Fernández, 2006).

Los cursos en los que se emplea un sentido del humor positivo suelen recibir mejores evaluaciones, en general, y los estudiantes suelen tener la percepción de haber aprendido más (Jáuregui Narváez \& Fernández Solís, 2009).

Según Idígoras (2002) el sentido del humor se considera un elemento didáctico con diferentes efectos en el contexto educativo:

- $\quad$ motiva el aprendizaje de los estudiantes;

- $\quad$ los predispone en una inmejorable actitud 
positiva;

- $\quad$ posibilita un clima distendido y agradable;

- $\quad$ favorece la empatía del educador;

- $\quad$ potencia la simpatía docente;

- facilita nuevas herramientas de trabajo; y,

- $\quad$ ejercita destrezas y habilidades creativas de los docentes.

Asimismo, Freire (2004) sostiene que el humor proporciona un ambiente en el cual el proceso de enseñanza-aprendizaje fluiría de forma más agradable, con menos resistencias por parte de ambos actores institucionales. Sin embargo, no hay estudios empíricos que confirmen estas hipótesis.

La empatía y sentido del humor tienen características distintivas; no obstante, el uso de un humor benéfico asociado a la empatía puede colaborar a generar un clima educativo propicio para el aprendizaje y promover conductas prosociales en los estudiantes.

Dentro de nuestro conocimiento, existe un único trabajo que ha relacionado la empatía y el humor. En esa investigación, Hampes (2010) encontró en una muestra intencional de 103 estudiantes de EE UU de 18 a 56 años que las personas que emplean un humor agresivo tienden a puntuar bajo en empatía.

Esto sugeriría que aquellos que utilizan el humor agresivo no sólo no quieren ayudar a alguien que se siente mal, sino que tienen dificultades para experimentar, tanto emocional y cognitivamente, los sentimientos negativos de la persona. A su vez, hallaron una fuerte correlación entre el humor agresivo y la toma de perspectiva.

Es interesante este resultado ya que es difícil imaginar cómo alguien que tiene dificultad para entender la perspectiva de otra persona sentiría simpatía o angustia en la situación de la misma.

Además, el humor de afiliación interpersonal aparentemente estaría más fuertemente relacionado con la preocupación empática "emocional" que el humor descalificación personal, así como el humor de mejoramiento personal es más intrapersonal y aparentemente estaría más fuertemente relacionada con la empatía de toma de perspectiva "cognitiva" que el humor de afiliación.

El autor explica que esto es consistente con Davis (1983), quien encontró que de los dos tipos de empatía, la preocupación empática es una medida más "emocional" de empatía y que la adopción de perspectiva, es más "cognitiva".

Aún no existen investigaciones que hayan analizado la relación entre los niveles de empatía de docentes primarios y los 4 tipos de humor propuestos por Martín et al., (2003).

El objetivo de este trabajo es evaluar esas relaciones y compararlos con las halladas por Hampes. Se hipotetiza que los estilos de humor adaptativos predecirán la adopción de perspectiva, alegría empática y comprensión empática, por el contrario, el humor descalificación predecirá el estrés empático.

\section{Método}

Participantes: La muestra fue no probabilística y se encontró conformada por 84 docentes mujeres de entre 23 y 49 años de edad pertenecientes a escuelas públicas del nivel primario (de $1^{\circ}$ a $6^{\circ}$ año) de la Región 
educativa $\mathrm{n}^{\circ}$ IV de la provincia de Buenos Aires, Argentina (incluye las localidades de: Quilmes, Berazategui y Florencio Varela).

Dichas escuelas están compuestas por una comunidad educativa bastante homogénea pertenecientes a un nivel socio-económico medio-bajo, de clase trabajadora. Las profesionales cuentan con uno o dos cargos docentes con una carga horaria de entre 20 y 40 horas semanales.

La muestra se dividió en dos grupos según las edades de acuerdo a la clasificación de Mansilla (2000) en su trabajo "Etapas del desarrollo humano", en el cual define la categoría "Adulto" como todo ser humano desde los 25 a los 64 años de edad.

Dividimos la muestra en dos primeros grupos entre 25 a 49 años. Dado que hay muy pocos docentes trabajando con más de 50 años: 1) adultos Jóvenes o adultos primarios (25 a 39 años, $n=49$, Media=33,41, DS=5,1, $\mathrm{Me}=34)$.

Son personas que cuentan con un trabajo y una familia en crecimiento. Enfrenta la madurez de su desarrollo; 2) adultos o adultos intermedios (40 a 49 años, n=35, Media=44, $\mathrm{DS}=2.85, \mathrm{Me}=44)$. En este grupo predomina el enfrentamiento a cambios familiares y entre parejas.

Instrumentos:CuestionarioSociodemográfico, incluye edad, estado civil, cantidad de cargos, antigüedad en el puesto, Cuestionario de Estilos del Humor, se administró el instrumento Humor Styles Questionnaire (HSQ) creado por Martin et al., (2003) y adaptado el castellano y validado en población argentina (Cayssials
\& Perez, 2005). Esta escala evalúa las diferencias individuales en los cuatro estilos de humor (dos adaptativos y dos mal adaptativos).

El instrumento está compuesto por 29 ítems con una escala tipo Likert que mide el rango de acuerdo (uno, totalmente en desacuerdo a cinco, totalmente de acuerdo); a mayor puntaje mayor humor.

Todos los ítems fueron traducidos $\mathrm{y}$ administrados a una muestra piloto de población general de Argentina. Luego se llevó a cabo su adaptación lingüística definitiva (Cayssials, 2004).

En el estudio de Cayssials y Perez, (2005) se corroboró la validez de la técnica a partir del análisis de su estructura factorial. El análisis de los componentes principales (Varimax) arrojó cuatro factores correspondientes a las cuatro escalas del cuestionario y obtuvo un Alpha general de 0.75.

El alfa de la presente muestra de todo el cuestionario fue de 0.83 , un poco más alto que la del original. A continuación se presentan las 4 escalas con algún ejemplo, los datos del número de ítems que contiene, la consistencia interna del instrumento original y de la presente muestra.

\section{Humores Adaptativos}

Afiliativo (AF, nueve ítems; alfa de Cronbach= 0.73 y 0.72 en la presente muestra). Ejemplo de ítem: "Me gusta reírme o hacer bromas con la gente").

Mejoramiento personal (MP, ocho ítems, alfa de Cronbach $=0.75$ y 0.74 en la presente muestra. Ej. "Si estoy triste, el humor me ayuda a levantar mi estado de ánimo").

\section{Humores Desadaptativos}

Agresividad (AG, ocho ítems, alfa de Cronbach= 0.80 y 0.25 en la presente muestra). Ej. "Si alguien 
se equivoca, trato de "gastarlo" o hacerle una cargada").

Descalificación personal (DP,ocho ítems, alfa de Cronbach $=0.73$ y 0.75 en la presente muestra). Ej. "Dejo que me hagan bromas o chistes más de lo necesario").

Test de empatía cognitiva y afectiva (TECA). Este cuestionario evalúa, desde una aproximación cognitiva y afectiva, la capacidad de los adultos de interactuar de manera eficaz con el mundo social.

El test fue creado por López- Pérez, Fernández- Pinto y Abad (2008) y es el único de este grupo que fue elaborado en español originalmente. El test se encuentra desarrollado para ser utilizado en adultos (Oceja, López-Pérez, Ambrona, \& Fernández, 2009).

Es una escala de autoinforme con 33 items mediante una escala Likert evalúa la empatía cognitiva y emocional con un rango de cinco puntos que van de uno (total desacuerdo) a cinco (muy de acuerdo) donde a mayor puntaje mayor empatía. En esta investigación la palabra "personas" de los enunciados fueron modificados por "alumnos" ya que su finalidad fue la apreciación de la capacidad empática desde una aproximación cognitiva y afectiva de los docentes hacia los alumnos.

Evalúa una dimensión global de empatía y cuatro escalas específicas, los valores de consistencia interna de este test, según informa el estadístico alfa de Cronbach, son de 0.86 en el caso de la puntuación global y superior a 0.70 en todas las escalas.

A continuación de presentan la dimensiones, número de ítems, la consistencia interna de la presente muestra, su concepto y ejemplo.

Adopción de perspectivas (AP, ocho ítems, alfa de Cronbach=0.65).
Evalúa la capacidad para la tolerancia, la comunicación y las relaciones personales. Ej. "Cuando un alumno se ha portado mal conmigo intento entender sus motivos."

Comprensión emocional (CE, nueve ítems, alfa de Cronbach=0.44). Implica la capacidad para reconocer y comprender los estados emocionales de los otros; así como sus intenciones e impresiones;por ej. "Me doy cuenta cuando mis alumnos están especialmente contentos sin que me hayan contado el motivo".

Estrés empático (EE, ocho ítems, Alfa de Cronbach $=.68$ ). Se refiere a la conexión con los estados emocionales negativos de los otros; por ej. "Me afectan demasiado los problemas de mis alumnos."

Alegría empática (AE, ocho ítems, alfa de Cronbach $=0.58$ ) Se refiere a la capacidad para compartir las emociones positivas de otras personas; por ej. "Me encanta ver que un alumno nuevo se encuentra a gusto en el aula".

Procedimiento: Los instrumentos se han administrado durante los meses de junio y julio del ciclo lectivo 2017 en cada establecimiento educativo, previa autorización de cada Directivo.

Los cuestionarios fueron entregados en mano a cada docente junto con el consentimiento informado y con la correspondiente instrucción para ser completados. Los datos se han tratados con confidencialidad y anonimato de sus respuestas.

Las docentes tenían tres días para completar los cuestionarios en sus domicilios o en las horas libres institucionales para no interferir con la tarea educativa en el aula. 
Procedimiento estadístico: Los datos se analizaron con el programa estadístico Statistical Packge for the Social Science (SPSS.13). Se realizó un análisis descriptivo de los datos obtenidos con las evaluaciones, seguidamente se realizó un estudio de correlación entre las variables en estudio.

Posteriormente se realizó el análisis de regresión lineal con el objetivo de estudiar Tabla 1

Análisis descriptivo de los datos $(n=84)$.

\begin{tabular}{lll}
\hline Variables & Medias & Desvío Estándar \\
\hline Humor & & \\
AF & 28.55 & 5.5 \\
MP & 28.87 & 5.65 \\
AG & 16.76 & 3.43 \\
DP & 16.54 & 5.53 \\
Empatía & & \\
$\quad$ AD & 31.21 & 4.01 \\
CE & 33.05 & 3.77 \\
AE & 35.32 & 3.66 \\
EE & 26.19 & 4.84 \\
\hline
\end{tabular}

Relaciones entre estilos de humor y tipos de empatía

Los estudios de correlación indicaron que existe una correlación positiva entre el humor afiliativo y los distintos tipos de empatía, tanto emocional como cognitiva. Asimismo, indican que el humor descalificación personal presenta asociación con el estrés empático y el humor Mejoramiento personal con la empatía emocional: alegría empática. En cuanto al humor agresivo no encuentra asociaciones ni positivas ni negativas en relación a la empatía de las docentes de primaria (Tabla 2).

Tabla 2

Correlación entre los estilos de humor y los subtipos de empatía

\begin{tabular}{lllll}
\hline Estilos de HUMOR & AP & CE & AE & EE \\
\hline Afiliativo & $.287^{* *}$ & $.299^{* *}$ & $.311^{* *}$ & $.233^{*}$ \\
Mejoramiento personal & .173 & .029 & $.263^{*}$ & .144 \\
Agresivo & -.127 & .016 & .064 & .163 \\
Descalificación personal & -.001 & -.023 & .147 & $.403^{* *}$ \\
\hline
\end{tabular}

Nota: $* p<.05, \quad * * p<.01$. 
Predicción de los tipos de humor

$\mathrm{Se}$ realizaron cuatro análisis de regresión lineal con el objetivo de estudiar si los distintos tipos de empatía permiten predecir los tipos de humor.

En todos los casos las dimensiones de la variable empatía fueron utilizadas como VI y los tipos de humos como VD.

En relación al humor agresivo, los resultados indican que el modelo fue significativo $\mathrm{F}(4,79)=1.58, \mathrm{p} \leq .001$, la adopción de perspectiva inhibe el uso del humor agresivo $(\beta=-.261)$ no se encontró relación con las otras dimensiones de la empatía. Este modelo explicó el 10\% de la varianza $(\mathrm{R} 2=.10$, ver Tabla 3$)$.

Tabla 3

Predicción del Humor Agresivo

\begin{tabular}{llll}
\hline Variables predictivas & B & EE C & $\beta$ \\
\hline Adopción de perspectiva & -.219 & .109 & $-.261^{*}$ \\
Comprensión empática & .060 & .112 & .066 \\
Alegría empática & .083 & .119 & .088 \\
Estrés empático & .177 & .099 & .210 \\
\hline Nota: ${ }^{*} p<.05,{ }^{* * *} p<.001$ & & &
\end{tabular}

En relación al humor descalificación personal, los resultados indican que el modelo fue significativo $\mathrm{F}(4,79)=4.54, \mathrm{p} \leq .002$, el estrés empático predice positivamente el estilo de humor de descalificación personal $(\beta=$
.425) sin embargo no se encontró relación con los otras dimensiones de la empatía. Estos análisis de regresión explicaron el $19 \%$ de la varianza $(\mathrm{R} 2=.19)$. Ver tabla 4.

Tabla 4

Predicción del Humor Descalificación Personal

\begin{tabular}{llll}
\hline Variables predictivas & $\mathrm{B}$ & EE C & $\boldsymbol{\beta}$ \\
\hline Adopción de perspectiva & -.179 & .164 & -.133 \\
Comprensión empática & -.087 & .170 & -.059 \\
Alegría empática & .109 & .183 & .072 \\
Estrés empático & .484 & .126 & $424^{* *}$ \\
\hline
\end{tabular}

Nota: $* p<.05, \quad * * * p<.001$ 
Posteriormente se analizó la influencia de la empatía sobre el humos mejoramiento personal $(\mathrm{F}(4,79)=1.86, \mathrm{p}=.13)$ y humor afiliativo $(\mathrm{F}(4,79)=3.86, \mathrm{p}=.71)$ no se hallaron resultados significativos.

\section{Diferencias de los tipos de humor y empatia} según el grupo etario

Se han encontrado diferencias significativas en el humor mejoramiento personal $\mathrm{F}(1,82)=4.60 ; \mathrm{p}<.035$, siendo superior en el grupo de mujeres con edad intermedia. En cuanto a los otros estilos de humor y las escalas de empatía no se han encontrado resultados significativos.

\section{Discusión}

La relevancia del estudio se centra en evaluar la posible existencia de una asociación entre los constructos teóricos: empatía y humor en docentes de primaria, ya que hasta el momento no se han encontrado estudios empíricos que vinculen ambas cualidades en esta clase de muestra.

Los resultados obtenidos mostraron una correlación positiva y significativa aunque no muy robusto, entre el humor afiliativo con los cuatro subtipos de empatía, el humor mejoramiento personal vinculado a la alegría empática y el humor descalificación personal con el estrés empático.

De manera que estos resultados encuentran que los estilos de humor afiliativo (interpersonal), están vinculado a la habilidad empática tanto cognitiva como emocional. En cuantoalhumoragresivo,comoeradeesperarse, no posee relaciones positivas significativas con la empatía. En cambio, los humores intrapersonales: adaptativo (mejoramiento personal) y desadaptivo (descalificación personal) se han asociado a la empatía emocional. Por ejemplo, el humor descalificación personal, humor hacia uno mismo en el que prevalece el autodesprecio, está asociado positivamente con el estrés emocional y malestar ante el dolor ajeno.

La relación entre ambas características coincide en parte con los resultados de Hampes (2010) hecho en EEUU con una población de estudiantes de psicología.

Al igual que en nuestra investigación, Hampes obtuvo correlaciones bajas aunque significativas en los humores adaptativos con los aspectos cognitivos y emocionales de la empatía, pero halló relaciones inversas con el humor agresivo, situación que no sucedió con los resultados de la presente investigación.

Estas comparaciones hay que tomarlas con precaución porque Hampes tomó los mismos constructos teóricos pero utilizando otros instrumentos y el muestreo de ambas publicaciones fue intencional, disminuyendo el poder de generalización.

Es importante también tener en cuenta que una de las limitaciones que presenta este estudio es el nivel bajo de fiabilidad de algunas de las dimensiones de los instrumentos utilizados para evaluar las varaibles..

Por otro lado, esta investigación está realizada con docentes mujeres, debido a que en la Argentina el 76\% de los docentes de primaria son mujeres (CEMPE, 2014). En consecuencia, no es claro cómo se presenta y relaciona la empatía y los estilos de humor en el género masculino. 
Cabe destacar que tanto el sentido de humor afiliativo como el agresivo se focalizan en el uso del humor en la relación interpersonal, mientras que el mejoramiento personal y el estilo de descalificación personal se centran en el uso del humor hacia uno mismo.

Es decir, que se pueden clasificar estos estilos de acuerdo a cómo se expresa el humor (a los demás o a sí mismo). Así, de los cuatro estilos de humor, dos aluden al mismo como una actividad relacional y los otros dos estilos hacen referencia al sentido del humor como una actividad intrapsíquica (Martin, 2003; Kuiper, Grimshaw, Leite \& Kirsh, 2004).

A partir de los resultados obtenidos en las correlaciones, pareció apropiado realizar un análisis de regresión lineal con el objetivo de estudiar el poder predictivo de los distintos tipos de empatía sobre cada uno de los tipos de humor.

En relación a la predicción de los diferentes tipos de empatía sobre los subtipos de humor, se obtuvo una varianza explicada de $10 \%$, con el humor agresivo a partir de una regresión negativa con la adopción de perspectiva y una varianza explicada del 19\% con el humor descalificación personal donde el estrés empático lo predice.

De manera que la predicción del estrés empático sobre el tipo de humor descalificativo, nos informa sobre la importancia del procesamiento del malestar ante el dolor ajeno en relación al humor descalificativo hacia uno mismo, aunque el poder predictivo resultó bajo.

Según Spreng, McKinnon, Mar y Levine (2009) la escala de estrés empático mide en gran medida las molestias, la ansiedad y el miedo a perder el control en situaciones amenazantes en general, en lugar de simplemente angustiarse en situaciones interpersonales.

En consecuencia, tanto el humor descalificación personal, vinculado más a la relación con uno mismo, y el estrés empático, afín a las sensaciones personales de malestar a emociones negativas ajenas, estarían asociados a la sensación de pérdida de control en situaciones desafiantes y por ende el uso del humor descalificativo, podría ser un predictor de un manejo más ansioso ante las situaciones angustiantes que le suceden a los otros.

Los resultados brindarían cierta evidencia de estas relaciones, sumado a que el estrés empático predice el tipo de humor descalificativo.

Por el contrario, el mejoramiento personal se encuentra vinculado a la alegría empática, tanto ese estilo de humor como la alegría empática comparten el reconocimiento de emociones propias $y$ ajenas, por ej. dicho humor se usa como una estrategia de afrontamiento para regular las emociones negativas y afrontarlas de forma que la persona no se dañe a sí mima ni a otros (Hampes, 2010).

En relación al rango etario, el grupo de mujeres en edad intermedia (40 a 49 años) han obtenido mayor humor mejoramiento personal.

Este resultado podría deberse a que las docentes con mayor edad habrían desarrollado mayores niveles de resiliencia. Tal como afirma Bobek (2002) el humor es vital para fortalecer la resiliencia de un maestro.

Mediante el uso del humor apropiado, los docentes pueden explorar alternativas para 
resolver problemas de una manera menos amenazante. En general este tipo de humor permite disminuir emociones negativas $\mathrm{y}$ mantener visiones positivas $\mathrm{y}$ realistas en situaciones adversas.

Posee una relación inversa con emociones negativas como ansiedad, depresión y, en general, con el neuroticismo (Martin et al., 2003). De manera que el humor sirve de catarsis emocional para los profesores y disminuye el cansancio físico y mental del proceso de enseñar (Freire, 2004).

Bobek (2002) afirma que los buenos maestros poseen altos niveles de competencia, toma de decisiones adecuadas y humor apropiado para crear ambientes de clase que estimulen el aprendizaje y enfaticen el rendimiento.

Entonces para generar un ambiente agradable y promover mayor adherencia en la curricula por parte de los alumnos probablemente sea un predictor el uso adecuado del humor y la empatía.

Otro aspecto a reflexionar, es el contexto institucional. El humor fomentaría un aumento de la calidad socioemocional de los maestros y por ende según estudios previos la satisfacción laboral de los docentes promueve una mejora en el aprendizaje de los alumnos (Allen, Witt \& Wheeless, 2006; Caprara, Barbaranelli, Steca \& Malone, 2006).

Hurren (2006) ha encontrado una fuerte relación entre el humor de los directores y la satisfacción laboral de los maestros, Por lo tanto, existe la posibilidad de que los logros de los estudiantes mejoren a medida que entre los colegas compartan más interacciones basadas en el humor. Es una característica que podría estudiarse en otros estudios, con el objetivo de analizar de manera integral las redes sociales en el ámbito escolar.

Si bien la presente investigación tiene un objetivo teórico, ella puede dar inicio a próximos estudios que indaguen las influencias del humor y la empatía de los docentes sobre parámetros educativos, como en el clima educativo o en el rendimiento académico de los alumnos.

Por ejemplo, en una investigación experimental, se realizaron dos tipos de clases: una basada en la enseñanza utilizando un estilo humorístico y el otro grupo en ausencia de expresiones de humor.

Los resultados informaron que el grupo que estudiaba con humor por parte de los docentes, obtuvo puntajes más altos en el examen final (Ziv, 1988).

Entonces, en futuros estudios podrían incluirse las calificaciones de los alumnos o reportes de los estudiantes acerca de las conductas empáticas y del uso del humor de sus maestros.

Para finalizar, en consonancia con Pérez (2011) cualquiera sea el estilo empático predominante en un profesional de la educación, lo importante es que mantenga un equilibrio entre la vertiente cognitiva y la afectiva, así como que preste atención a la "distancia educativa óptima".

Además de la empatía de los docentes, según nuestros resultados, el uso del humor benéfico, en especial el afiliativo, en el aula se encuentra relacionada con la empatía cognitiva y emocional de las docentes. 


\section{Referencias}

Alemany, C. \& R. Cabestrero (2008). Humor y psicoterapia: estudios e investigaciones. En A. R, Idígoras (ed.). El valor terapéutico del humor (pp. 111-186). Bilbao: Desclée De Brouwer,

Allen, M., Witt, P. L., \& Wheeless, L. R. (2006). The role of teacher immediacy as a motivational factor in student learning: Using meta-analysis to test a causal model. Communication education, 55(1), 21-31.

Askildson, L. (2005). Effects of humor in the language classroom: Humor as a pedagogical tool in theory and practice. The Arizona Working Papers in Second Language Acquisition and Teaching, 12, 45-61.

Bobek, B. L. (2002). Teacher resiliency: A key to career longevity. The Clearing House, 75(4), 202-205.

Camacho, J. M. (2005). El humor en la práctica de la psicoterapia de orientación sistémica (Doctoral dissertation, Tesis doctoral no publicada). Buenos Aires, Universidad de Buenos Aires).

Caprara, G. V., Barbaranelli, C., Steca, P., \& Malone, P. S. (2006). Teachers' self-efficacy beliefs as determinants of job satisfaction and students' academic achievement: A study at the school level. Journal of school psychology, 44(6), 473-490.

Carkhuff, R. R., \& Berenson, B. (1967). Beyond counseling and therapy. New York: Holt, Rinehart, \& Winston.
Cayssials, A. (2004): Ficha de la cátedra. Sentidos del Humor. Dpto. de Publicaciones. Facultad de Psicología. UBA

Cayssials, A. N., \& Pérez, M. A. (2005). Características psicométricas del cuestionario de estilos del humor (HSQ). Un estudio con población argentina. XII Jornadas de Investigación de la Facultad de Psicología de la Universidad de Buenos Aires, 4(5).

CENPE (2014). Censo Nacional del Personal de los Establecimientos Educativos. Ministerio de Educación y Deportes, Argentina.

Chang, L. (2003). Variable effects of children's aggression, social withdrawal, and prosocial leadership as functions of teacher beliefs and behaviors. Child development, 74(2), 535-548.

Davis, M. H. (1980). A multidimensional Approach to Individual Differences in Empathy. JSAS Catalog of Selected Documents in Psychology, 10, 85.

Davis, M. H. (1983). Measuring individual differences in empathy: Evidence for a multidimensional approach. Journal of personality and social psychology, 44(1), 113.

Decety \& W. Ickes (Eds.), The social neuroscience of empathy (pp. 3-15). Cambridge, MA: MIT Press.

Durán, A., Extremera, N., Montalbán, F. \& 
Rey, L.(2005). Engagement y Burnout en el ámbito docente: Análisis de sus relaciones con la satisfacción laboral y vital en una muestra de profesores. Revista de Psicología del Trabajo y las Organizaciones, 21, 145-158.

Eisenberg, N., \& Strayer, J. (1987). Critical issues in the study of empathy. In N. Eisenberg \& J. Strayer (Eds.), Empathy and its development (pp. 3-13). New York: Cambridge University Press

Eysenck, H. J. (1942). The appreciation of humour: an experimental and theoretical study 1. British Journal of Psychology. General Section, 32(4), 295-309.

Freire, J. B. (2004). El sentido del humor en el aula. XXXII Jornadas de Centros Educativos: Una comunicación de puertas abiertas.

Freire, P. (2004). Pedagogía de la Autonomía. Saberes necesarios para la práctica educativa. México. Siglo XXI.

Gleichgerrcht, E. y Decety, J. (2013). Empathy in Clinical Practice:How Individual Dispositions, Gender, and Experience Moderate Empathic Concern, Burnout, and Emotional Distress in Physicians. Plos One 2013; 8(4): e61526 doi: 10.1371/journal.pone.0061526.

Fitriah, F. (2017). Appropriate and inappropriate uses of humor by teachers and the effect of it in learning. LET: Linguistics, Literature and English Teaching Journal, 2(2), 114-120.
Hampes, W. P. (2010). The relation between humor styles and empathy. Europe's Journal of Psychology, 6(3), 34-45.

Hatfield, E., Cacioppo, J. T., \& Rapson, R. L. (1994). Emotional contagion: Cambridge studies in emotion and social interaction. Cambridge, UK: Cambridge University Press. errors-in-variables regression model when the variances of the measurement errors vary between the observations. Statistics in Medicine, 21, 1089-1101.

Hurren, B. L. (2006). The effects of principals' humor on teachers' job satisfaction. Educational Studies, 32(4), 373-385.

Illán, J. (2015). El uso del humor en terapia. WWW.DONPSICO.ES Recuperado de: https://psicocode.com/clinica/el-uso-delhumor-en-terapia/, el 20/11/2017.

Jáuregui, E. y Fernández Solís, J. D. (2006). E1 humor positivo en la vida y el trabajo. CÍRCULO de Lingüística Aplicada a la Comunicación (clac), 27, 42-56.

Jáuregui Narváez, E., \& Fernández Solís, J. D. (2009). Risa y aprendizaje: el papel del humor en la labor docente. Revista interuniversitaria de formación del profesorado, 23(3).

Kuiper, N. A., Grimshaw, M., Leite, C., \& Kirsh, G. (2004). Humor is not always the best medicine: Specific components of sense of humor and psychological well-being. Humor: International Journal of Humor Research., 17, 1-2. 
Lefcourt, H. M., Davidson, K., Shepherd, R., Phillips, M., Prkachin, K., \& Mills, D. (1995). Perspective-taking humor: Accounting for stress moderation. Journal of Social and Clinical Psychology, 14(4), 373-391.

López-Pérez, B., Fernández-Pinto, I., \& García, F. A. (2008). TECA: test de empatía cognitiva $y$ afectiva: manual. TEA.

Martin, R. A., Puhlik-Doris, P., Larsen, G., Gray, J., \& Weir, K. (2003). Individual differences in uses of humor and their relation to psychological well-being: Development of the Humor Styles Questionnaire. Journal of research in personality, 37(1), 48-75.

Menghi, M. S., \& Oros, L. B. (2014). Satisfacción laboral y Síndrome de Burnout en docentes de nivel primario. Revista de Psicología, 10 (20), 47-59.

Moya-Albiol, L., Herrero, N., \& Bernal, M. C. (2010). Bases neuronales de la empatía. Revista Neurol, 50(2), 89-100.

Oceja, L., López-Pérez, B., Ambrona, T., \& Fernández, I. (2009). Measuring general dispositions to feeling empathy and distress. Psicothema, 21(2), 171-176.

Pérez, V. M. O. (2011). La empatía en la educación: estudio de una muestra de alumnos universitarios. Revista Electrónica de Psicología Iztacala, 14(4), 174.

Pianta, R. C., La Paro, K. M., \& Hamre, B. K. (2008). Classroom Assessment Scoring System ${ }^{\text {TM}: ~ M a n u a l ~ K-3 . ~ P a u l ~ H ~ B r o o k e s ~}$ Publishing.
Pollak, J. P. \& Freda, P.D. (1997). Humor, learning, and socialization in middle level classrooms. The Clearing House 70(4), 176-78.

Richaud, M. C. (2014). Algunos aportes sobre la importancia de la empatía y la prosocialidad en el desarrollo humano. Revista Mexicana de Investigación en Psicología, 6(2)

Rodríguez, A. (2002). La dimensión terapéutica del humor. En A. Rodríguez (ed.). El valor terapéutico del humor (pp. 15-40).Bilbao: Desclée de Brouwer

Seligman, M. E. P. (2007). La Autentica Felicidad/AuthenticHappiness (Spanish Edition). Ediciones B.

Spreng, R. N., McKinnon, M. C., Mar, R. A., \& Levine, B. (2009). The Toronto Empathy Questionnaire: Scale development and initial validation of a factor-analytic solution to multiple empathy measures. Journal of personality assessment, 91(1), 62-71.

Ziv, A. (1988). Teaching and learning with humor: Experiment and replication. The Journal of Experimental Education, 57(1), 4-15. 